\title{
Legendre-Galerkin method for nonlinear partial \\ differential equations
}

\author{
Jun Liu ${ }^{\mathrm{a}}$, XinYue Fan \\ ${ }^{a, b}$ Department of Computer science, \\ School of mathematics and statistics, Guizhou University, Guiyang, China \\ a597935697@qq.com, ${ }^{\text {b fan.xinyue@163.com }}$
}

\section{Keywords: Legendre-Galerkin method; Burgers equation; Error estimate}

Abstract. In this paper, a Legendre-Galerkin method is proposed and analyzed for the Burgers equation with dirichlet boundary condition. We present in this paper the error estimation concerning Legendre approximations in Sobolev spaces, in which integration is performed with respect to the Legendre weight $\omega(x)=1$. It is shown that the Legendre-Galerkin approximations are convergent on the interval $[-1,1]$ with spectral accuracy. An efficient and accurate algorithm based on the Legendre-Galerkin approximations to the Burgers equation is developed and implemented. Finally the numerical results which indicate that the high accuracy and effectiveness of this algorithm are presented.

\section{Introduction}

The Legendre-Spectral approximations for linear partial differential equation have achieved great success and popularity in the past decades, Pioneer work on Legendre-Galerkin approximation was developed in ShenJie and TangTao [1] , and GuoBenYu [2], CHQZ [3,4], see also Maday [5]. From the point of numerical calculation, nonlinear Galerkin method can be used as a space decomposition method, the solution subspace decomposition as low modulus components of tensor subspace and anti-embrittlement tensor subspace, and the solution of equation respectively to the two subspace projection, due to the anti-embrittlement component part is small, so it can be more flexible in terms of processing, so as to achieve the purpose of reduce amount of calculation, in the actual calculation can be used in a variety of methods, spectrum and spectrum method is a powerful tool, and the discussion of inertial manifolds is established on the basis of space according to the spectral decomposition. Based on one dimensional nonlinear Galerkin Buregers equation - Legendre spectral method to construct the format, then discuss its stability, convergence and error estimates. The aim of this paper is to develop a Legendre-Galerkin approximation and to construct an efficient, accurate and stable numerical algorithm for the Burgers equation on the interval [-1,1].

First of all, we consider the following Burgers equation with Dirichlet boundary condition

$$
u_{t}-v \frac{\partial^{2} u}{\partial x^{2}}+u \frac{\partial u}{\partial x}=0
$$

for $x \in \Omega$ and $t \in[0, T]$ where $\Omega$ is a bounded domain in [-1,1]. Here the viscous coefficientv $>0$ with the initial condition 


$$
u(x, 0)=u_{0}(x)
$$

and the boundary condition

$$
u( \pm 1, t)=0
$$

The Burgers equation, it retains the nonlinear aspects of the governing equation in many practical transport problems such as aggregation interface growth, shock wave theory, transport and dispersion of pollutants in atmosphere and so on.

The rest of the paper is organized as follows. In section 2, we provide some basic conclusion on Legendre approximations. In section 3, we propose a suitable Legendre-Galerkin approximation for Burgers equation. In section 4, we present the stability and error estimates. In section 5, we construct efficient algorithms for the Burgers equation, and present some numerical results.

\section{Some basic formulas on Legendre polynomials}

The Legendre polynomial of degree $\mathrm{n}$ is defined by

$$
L_{n}(x)=\frac{1}{2^{n}} \sum_{l=0}^{\frac{n}{2}}(-1)^{n}\left(\begin{array}{l}
n \\
l
\end{array}\right)\left(\begin{array}{l}
2 n-2 l \\
n
\end{array}\right) x^{n-2 l}
$$

The Legendre polynomials $L_{n}(x)$ are generated from the singular Sturm-Liouville problem with $\omega(x) \equiv 1,(a, b)=(-1,1)$ and the normalization $L_{n}(1)=1$. The Legendre polynomials satisfy the follow relation

$$
L_{0}(x)=1, \quad L_{1}(x)=x
$$

$(n+1) L_{n+1}(x)=(2 n+1) x L_{n}(x)-n L_{n-1}(x), \quad n \geq 1 \quad(2.3)$

and the orthogonality relation

$$
\int_{-1}^{1} L_{k}(x) L_{j}(x) d x=\frac{2}{2 k+1} \delta_{k j} .
$$

other useful properties of the Legendre polynomials include:

$$
\begin{gathered}
L_{n}^{\prime}(x)=\sum_{k=0}^{n-1}(2 k+1) L_{k}(x) \\
L_{n}^{\prime}(x)=\sum_{k=0}^{n-2}\left(k+\frac{1}{2}\right)(n(n+1)-k(k+1)) L_{k}(x)
\end{gathered}
$$

For the Legendre series, the quadrature points and weights are as the follows:

Legendre-Gauss: $x_{j}$ are the zeros of $L_{N+1}(x)$, and

$$
\omega_{j}=\frac{2}{\left(1-x_{j}^{2}\right)\left[L_{N+1}^{\prime}\left(x_{j}\right)\right]^{2}}, \quad 0 \leq j \leq N
$$

Legendre-Gauss-Radau: $x_{j}$ are the $\mathrm{N}+1$ zeros of $L_{N}(x)+L_{N+1}(x)$, and 
$\omega_{0}=\frac{2}{(N+1)^{2}}, \quad \omega_{j}=\frac{1}{(N+1)^{2}} \frac{1-x_{j}}{\left[L_{N}\left(x_{j}\right)\right]^{2}}, \quad 1 \leq j \leq N$.

Legendre-Gauss-Lobatto: $x_{0}=-1, x_{N}=1,\left\{x_{j}\right\}_{j=1}^{N-1}$ are the zeros of $L^{\prime}{ }_{N}(x)$, and

$$
\omega_{j}=\frac{2}{N(N+1)} \frac{1}{\left[L_{N}^{\prime}\left(x_{j}\right)\right]^{2}}, \quad 0 \leq j \leq N
$$

\section{Legendre-Galerkin method for the Burgers equation}

First we import some notations, setting as follows:

$$
\begin{gathered}
S_{N}=\operatorname{span}\left\{L_{0}(x), L_{1}(x), \mathrm{L}, L_{N}(x)\right\}, V_{N}=\left\{v \in S_{N} \mid v( \pm 1)=0\right\} . \\
Q_{2 N}=\operatorname{span}\left\{L_{N-1}(x), L_{N}(x), \mathrm{L}, L_{2 N}(x)\right\}, W_{2 N}=\left\{\omega \in Q_{2 N} \mid \omega( \pm 1)=0\right\} . \\
Q_{2 N} \oplus S_{N-2}=S_{2 N}=\operatorname{span}\left\{L_{0}(x), L_{1}(x), \mathrm{L}, L_{2 N}(x)\right\} . \\
V_{N} \oplus W_{2 N}=V_{2 N}=\left\{v \in S_{2 N} \mid v( \pm 1)=0\right\} .
\end{gathered}
$$

Note $P_{N}$ is $L^{2}(I) \rightarrow S_{N}$ quadrature projection operator, $I=(-1,1)$, i.e.

$$
\left(u-P_{N} u, v\right)=0, \quad \forall v \in S_{N}, u \in L^{2}(I) .
$$

Note $\prod_{N}$ is $H_{0}^{1}(I) \rightarrow V_{N}$ projection operator

$$
a\left(u-\prod_{N} u, v\right)=0, \quad \forall v \in V_{N}, u \in H_{0}^{1}(I) .
$$

where $a(u, v)=\int_{I} u_{x} v_{x} d x$.

Now we consider the following nonlinear equation

$$
\begin{gathered}
\frac{\partial u}{\partial t}-v u_{x x}+B(u)=f, \quad(x, t) \in I \times(0, T) \\
u(x, 0)=u_{0}(x) \\
u( \pm 1, t)=0 .
\end{gathered}
$$

Where $B(u)=B(u, u), \quad B(\cdot, \cdot)$ is the bilinear form, $b(u, v, \omega)=(B(u, v), \omega)$, and satisfies

$$
b(u, v, v)=0, \quad \forall v \in H_{0}^{1}(I)=V .
$$

and

$b(u, v, \omega) \leq C\|u\|_{V}\|v\|_{V}\|\omega\|_{V}, \quad \forall u, v \in H_{0}^{1}(I), \omega \in L^{2}(I)$.

We assume $B(u)=u u_{x}, B(u, v)=\frac{2}{3} u v_{x}+\frac{1}{3} v u_{x},(3.7)-(3.9)$ is a one-dimension Burgers equation 


$$
\begin{gathered}
\frac{\partial u}{\partial t}-v u_{x x}+u u_{x}=f, \quad(x, t) \in I \times(0, T) \\
u(x, 0)=u_{0}(x) \\
u( \pm 1, t)=0 .
\end{gathered}
$$

Its weak formulation is

$$
\begin{gathered}
\frac{d}{d t}(u, v)+v a(u, v)+b(u, u, v)=(f, v), \quad \forall v \in H_{0}^{1}(I), t \in(0, T] \\
u(x, 0)=u_{0}(x)
\end{gathered}
$$

The result of (3.10) from the definition and by integration by parts,

\section{Proof. Since}

$$
\begin{aligned}
& b(u, \omega, v)=(B(u, \omega), v)=(B(u, v), \omega)=\left(\frac{2}{3} u v_{x}, \omega\right)+\left(\frac{1}{3} v u_{x}, \omega\right) \\
& =\left.\frac{2}{3} u \omega v\right|_{\partial I}-\frac{2}{3} \int_{I}(u \omega)^{\prime} v d x+\left.\frac{1}{3} u \omega v\right|_{\partial I}-\frac{1}{3} \int_{I}\left(v^{\prime} u \omega+v \omega^{\prime} u\right) d x \\
& =-\frac{2}{3} \int_{I} u^{\prime} \omega v d x-\int_{I} u \omega^{\prime} v d x-\frac{1}{3} \int_{I} v^{\prime} u \omega d x
\end{aligned}
$$

Denote

$$
\begin{array}{r}
b 1(u, \omega, v)=-\frac{2}{3} \int_{-1}^{1} \omega(x) v(x) \frac{d u}{d x} d x-\int_{-1}^{1} u(x) v(x) \frac{d \omega}{d x} d x \\
b 2(u, \omega, v)=-\frac{1}{3} \int_{-1}^{1} \omega(x) u(x) \frac{d v}{d x} d x \\
b(u, \omega, v)=b 1(u, \omega, v)+b 2(u, \omega, v)
\end{array}
$$

when $\omega=v$,

$$
\begin{aligned}
b(u, \omega, v) & =-\frac{2}{3} \int_{-1}^{1} v^{2} \frac{d u}{d x} d x-\frac{4}{3} \int_{-1}^{1} u v \frac{d v}{d x} d x \\
& =-\frac{2}{3} \int_{-1}^{1} v^{2} \frac{d u}{d x} d x+\frac{4}{3} \int_{-1}^{1}(u v)^{\prime} v d x \\
=-\frac{2}{3} \int_{-1}^{1} v^{2} \frac{d u}{d x} d x+\frac{4}{3} \int_{-1}^{1} u^{\prime} v^{2} d x+\frac{4}{3} \int_{-1}^{1} u^{\prime} v^{\prime} v d x & \\
& =\frac{2}{3} \int_{-1}^{1} v^{2} u^{\prime} d x+\frac{4}{3} \int_{-1}^{1} u v^{\prime} v d x \\
& =-\frac{4}{3} \int_{-1}^{1} u v^{\prime} v d x+\frac{4}{3} \int_{-1}^{1} u v^{\prime} v d x
\end{aligned}
$$

$=0$

The result of (3.11) can be proofed with sobolev inequality and Young inequality, associative conclusion see [6].

Hence, we shall consider the following variational formulation of (3.12)-(3.14):

Find $u \in L^{2}\left(0, T ; H_{0}^{1}(I)\right)$ such that 


$$
\begin{gathered}
\frac{d}{d t}(u(t), \phi)+v a(u(t), \phi)+b(u(t), u(t), \phi)=(f(t), \phi), \quad \forall \phi \in V_{2 N}, t \in(0, T] \\
u_{2 N}(x, 0)=\prod_{2 N} u_{0}(x)
\end{gathered}
$$

The Legendre-Galerkin approximation for (3.29)-(3.30) is:

Find $u_{N}(t) \in V_{N}, u_{2 N}(t) \in W_{2 N}, \forall 0 \leq t \leq T$, such that

$$
\begin{gathered}
\frac{d}{d t}\left(u_{N}(t), \phi\right)+v a\left(u_{N}(t), \phi\right)+b\left(u_{N}(t)+u_{2 N}(t), u_{N}(t)+u_{2 N}(t), \phi\right)=(f, \phi), \\
\forall \phi \in V_{N}, t \in(0, T] \\
v a\left(u_{2 N}(t), \omega\right)+b\left(u_{N}(t)+u_{2 N}(t), u_{N}(t), \omega\right)=(f, \omega) \\
\forall \omega \in W_{2 N} \\
u_{N}(x, 0)=\prod_{N} u_{0}(x)
\end{gathered}
$$

Lemma 1. Let $u_{N}$ and $u_{2 N}$ be the solutions of (3.31)-(3.35). Then

$$
\begin{array}{r}
\left\|u_{N}(t)\right\|^{2}+v \int_{0}^{t}\|u(\tau)\|_{1}^{2} d \tau \leq C\left(\left\|u_{0}\right\|_{1}^{2}+\int_{0}^{T}\|f\|^{2} d s\right) \\
\forall t \in[0, T] \\
\forall t .36)
\end{array}
$$

Proof. By taking $\phi=u_{N}(t)$ in (3.31). By definition (3.10), we have

$$
\frac{1}{2} \frac{d}{d t}\left\|u_{N}(t)\right\|^{2}+v\|u(\tau)\|_{1}^{2}=\left(f, u_{N}(t)\right) \leq C\|f\|^{2}+\frac{v}{2}\|u(t)\|_{1}^{2}
$$

We conclude by applying the Gronwall lemma to the above inequality.

Remove $\frac{v}{2}\|u(t)\|_{1}^{2}$, then integral about $t$ in two sides.

Notice that $\left\|\Pi_{N} u_{0}\right\| \leq C\left\|u_{0}\right\|_{1}$, we have

$$
\begin{gathered}
\left\|u_{N}(t)\right\|^{2}+v \int_{0}^{t}\|u(\tau)\|_{1}^{2} d \tau \leq C\left(\left\|u_{0}\right\|_{1}^{2}+\int_{0}^{T}\|f\|^{2} d s\right) \\
\forall t \in[0, T] .
\end{gathered}
$$

\section{Error analysis in spacial scheme}

We now consider the error analysis for (3.31)-(3.35)

Theorem 1. With assume of (3.10) and (3.11), for all large N, we have

$$
\begin{aligned}
& \left(\int_{0}^{T}\left\|u(\tau)-u_{N}(\tau)-u_{2 N}(\tau)\right\|_{1}^{2} d \tau\right)^{\frac{1}{2}} \leq M(2 N)^{1-s}\|u\|_{L^{2}\left(0, T ; H^{s}\right)} \\
& +M N^{-s}\|u\|_{L^{2}\left(0, T ; H^{s}\right)}
\end{aligned}
$$




$$
+M N^{1-r}\left\|u_{t}\right\|_{L^{2}\left(0, T ; H^{r-2}\right)}
$$

Proof. Since $\prod_{2 N}$ defintion and satisfy

$$
\begin{gathered}
\frac{d}{d t}(u, \phi+\omega)+v\left(\left(\prod_{2 N} u\right)_{x},(\phi+\omega)_{x}\right)+b(u, u, \phi+\omega)=(f, \phi+\omega) \\
\forall \phi \in V_{N}, \omega \in W_{2 N} .
\end{gathered}
$$

Note $e=\prod_{2 N} u-\left(u_{N}+u_{2 N}\right), \xi=\prod_{N}-u_{N}, \eta=\left(\prod_{2 N}-\prod_{N}\right) u-u_{2 N}$

Where $\xi \in V_{N}, \eta \in W_{2 N}$, and $e=\xi+\eta$, especially

$$
\|e\|_{1}^{2}=\|\xi\|_{1}^{2}+\|\eta\|_{1}^{2}
$$

Then

$$
\begin{aligned}
& \frac{d}{d t}(u, \phi+\omega)-\frac{d}{d t}\left(u_{N}, \phi\right)+v a(e, \phi+\omega)+b(u, u, \phi) \\
& -b\left(u_{N}+u_{2 N}, u_{N}+u_{2 N}, \phi\right)+b(u, u, \omega)-b\left(u_{N}+u_{2 N}, u_{N}, \omega\right)=0
\end{aligned}
$$

Tidy it

$$
\begin{aligned}
& \qquad \frac{d}{d t}(u, \phi+\omega)-\frac{d}{d t}\left(u_{N}, \phi\right)+v a(e, \phi+\omega)+\left(u_{t}, \omega\right) \\
& =b\left(u_{N}+u_{2 N}, u_{N}+u_{2 N}, \phi\right)-b(u, u, \omega)+b\left(u_{N}+u_{2 N}, u_{N}, \omega\right)-b(u, u, \omega) \\
& \Rightarrow \quad \frac{d}{d t}\left(u-u_{N}+\prod_{N} u-\prod_{N} u, \phi\right)+v a(e, \phi+\omega) \\
& =-\left(u_{t}, \omega\right)+\left[b\left(u_{N}+u_{2 N}, u_{N}+u_{2 N}, \phi\right)-b(u, u, \phi)\right]+ \\
& \Rightarrow \quad=\left[b\left(u_{N}+u_{2 N}, u_{N}, \omega\right)-b(u, u, \omega)\right] \\
& =\left(\left(\prod_{N} u-u\right)_{t}, \phi\right)-\left(u_{t}, \omega\right)+\left[b\left(u_{N}+u_{2 N}, u_{N}+u_{2 N}, \phi\right)-b(u, u, \phi)\right]+ \\
& {\left[b\left(u_{N}+u_{2 N}, u_{N}, \omega\right)-b(u, u, \omega)\right]}
\end{aligned}
$$

Note $\phi=\xi$, and $\omega=\eta$, we have

$$
\begin{aligned}
& \frac{1}{2} \frac{d}{d t}\|\xi\|^{2}+v\|e\|_{1}^{2} \\
& =\left[\left(\left(\prod_{N} u-u\right)_{t}, \xi\right)-\left(u_{t}, \eta\right)\right]+\left[b\left(u_{N}+u_{2 N}, u_{N}+u_{2 N}, \xi\right)-b(u, u, \xi)\right]+ \\
& {\left[b\left(u_{N}+u_{2 N}, u_{N}, \eta\right)-b(u, u, \eta)\right]} \\
& =I 1+I 2+I 3
\end{aligned}
$$


Since $\eta \in W_{2 N}$,

$$
\begin{aligned}
& I 1=\left[\left(\left(\prod_{N} u-u\right)_{t}, \xi\right)-\left(\left(I-P_{N-2}\right) u_{t}, \eta\right)\right] \\
& \leq \frac{v}{8}\left(\|\xi\|_{1}^{2}+\|\eta\|_{1}^{2}\right)+C\left(\left\|\Pi_{N} u-u\right\|_{-1}^{2}+\left\|\left(I-P_{N-2}\right) u_{t}\right\|_{-1}^{2}\right) \\
& \leq \frac{v}{8}\|e\|_{1}^{2}+C\left(\left\|\Pi_{N} u-u\right\|_{-1}^{2}+\left\|\left(I-P_{N-2}\right) u_{t}\right\|_{-1}^{2}\right) \\
& I 2=b\left(u_{N}+u_{2 N}, u_{N}+u_{2 N}, \xi\right)-b(u, u, \xi) \\
& =-b\left(u_{N}+u_{2 N}, u_{N}-\prod_{2 N} u, \xi\right)-b\left(u_{N}+u_{2 N}, e, \xi\right)-b\left(u-\prod_{2 N} u+e, u, \xi\right) \\
& =I 21+I 22+I 23 \\
& I 3=b\left(u_{N}+u_{2 N}, u_{N}, \eta\right)-b(u, u, \eta) \\
& =-b\left(u_{N}+u_{2 N}, u_{N}-\prod_{2 N} u, \eta\right)-b\left(u_{N}+u_{2 N}, \xi, \eta\right)-b\left(u-\prod_{2 N} u+e, u, \eta\right) \\
& =I 31+I 32+I 33
\end{aligned}
$$

Notice that $I 22+I 32=0$. Applying Young inequality and (3.11)

$$
\begin{aligned}
& \begin{array}{r}
I 23+I 33=-b\left(u-\prod_{2 N} u+e, u, e\right) \\
\leq C\left(\left\|u-\prod_{2 N} u\right\|_{1}+\|e\|_{1}\right)\|u\|_{1}\|e\| \\
\leq \frac{v}{8}\|e\|_{1}^{2}+M\left(\left\|u-\prod_{2 N} u\right\|_{1}^{2}+\|e\|^{2}\right)
\end{array} \\
& \begin{aligned}
I 21+I 31=-b\left(u_{N}+u_{2 N}, u-\prod_{2 N} u, \xi\right)+b\left(u_{N}+u_{2 N}, u-\prod_{N} u, \eta\right) \\
=b\left(e-\prod_{2 N} u, u-\prod_{2 N} u, e\right)+b\left(e-\prod_{N} u, \prod_{2 N} u-\prod_{N} u, \eta\right)
\end{aligned} \\
& =E 1+E 2
\end{aligned}
$$

Since $\left\|\pi_{N}\right\|_{1} \leq C\|u\|_{1}$, applying Young inequality and (3.11)

$$
\begin{aligned}
& E 1 \leq C\left(\left\|\prod_{2 N} u\right\|_{1}+\|e\|_{1}\right)\left\|u-\prod_{2 N} u\right\|_{1}\|e\| \\
& \leq M\left\|u-\prod_{2 N} u\right\|_{1}\|e\|\left(1+\|e\|_{1}\right) \\
& \leq M\left(\left\|u-\prod_{2 N} u\right\|_{1}^{2}+\|e\|^{2}\right)+\frac{v}{8}\|e\|_{1}^{2}+C\left\|u-\prod_{2 N} u\right\|_{1}^{2}\|e\|^{2} \\
& \leq M\left(\left\|u-\prod_{2 N} u\right\|_{1}^{2}+\|e\|^{2}\right)+\frac{v}{8}\|e\|_{1}^{2}
\end{aligned}
$$

Applying Poincare inequality and (3.11) and $\|\eta\|_{1} \leq\|e\|_{1}$, for all $N$ large 


$$
\begin{aligned}
& E 2 \leq C\left(\left\|\Pi_{2 N} u\right\|_{1}+\|e\|_{1}\right)\left\|\Pi_{N} u-\Pi_{2 N} u\right\|_{1}\|\eta\| \\
& \leq M N^{-1}\left\|\Pi_{N}-\Pi_{2 N} u\right\|_{1}\|e\|_{1}+C N^{-1}\left\|\Pi_{N} u-\Pi_{2 N} u\right\|_{1}\|e\|_{1}^{2} \\
& \leq \frac{v}{8}\|e\|_{1}^{2}+M N^{-2}\left\|\Pi_{N} u-\prod_{2 N} u\right\|_{1}^{2}
\end{aligned}
$$

In conclusion, we have

$$
\begin{aligned}
& \frac{d}{d t}\|\xi\|^{2}+v\|e\|_{1}^{2} \leq M\|e\|^{2}+M N^{-2}\left\|\Pi_{N}-\prod_{2 N} u\right\|_{1}^{2}+(4.43) \\
& C\left(\left\|\Pi_{N} u-\prod_{2 N} u\right\|_{1}^{2}+\left\|\left(I-\prod_{N}\right) u_{t}\right\|_{-1}^{2}+\left\|\left(I-P_{N-2}\right) u_{t}\right\|_{-1}^{2}\right)
\end{aligned}
$$

Applying Gronwall inequality

$$
\begin{aligned}
& \|\xi(t)\|^{2}+\int_{0}^{t}\|e(\tau)\|_{1}^{2} d \tau \\
& \leq M N^{-2} \int_{0}^{t}\left(\left\|u-\prod_{N} u\right\|_{1}^{2}+\left\|u-\prod_{2 N} u\right\|_{1}^{2}\right) d \tau+M \int_{0}^{t}\left(\left\|u-\prod_{2 N} u\right\|_{1}^{2}+\right. \\
& \left.\left\|\left(I-\prod_{N}\right) u_{t}\right\|_{-1}^{2}+\left\|\left(I-P_{N-2}\right) u_{t}\right\|_{-1}^{2}\right) d \tau \\
& \leq M\left(N^{-2 s}+(2 N)^{2-2 s}\right) \int_{0}^{T}\|u\|_{s}^{2} d \tau+M N^{2-2 r} \int_{0}^{T}\left\|u_{t}\right\|_{r-2}^{2} d \tau
\end{aligned}
$$

Then

$$
\begin{aligned}
& \int_{0}^{T}\left\|\left(u-u_{N}-u_{2 N}\right)\right\|_{1}^{2} d \tau \\
& \leq \int_{0}^{T}\left(\left\|u-\prod_{2 N} u\right\|_{1}^{2}+M\left(N^{-2 s}+(2 N)^{2-2 s}\right) \int_{0}^{T}\|u\|_{s}^{2} d \tau+M N^{2-2 r} \int_{0}^{T}\left\|u_{t}\right\|_{r-2}^{2} d \tau\right. \\
& \leq M\left(N^{-2 s}+(2 N)^{2-2 s}\right) \int_{0}^{T}\|u\|_{s}^{2} d \tau+M N^{2-2 r} \int_{0}^{T}\left\|u_{t}\right\|_{r-2}^{2} d \tau
\end{aligned}
$$

\section{Efficient implementations and numerical results}

In this section, we consider Legendre-Galerkin approximation of Burgers equation. After discretizing it in time by using explicit Euler scheme, we need to solve, at each time step, a linear system of the form:

Find $u_{N} \in V_{N}$ such that

$$
\left(u_{t}, v\right)+v\left(u_{x}, v_{x}\right)+\left(u u_{x}, v\right)=0, \quad \forall v \in V_{N}
$$

Discrete in time:

$$
\left(\frac{u^{n}-u^{n-1}}{\Delta t}, v\right)+v\left(u_{x}^{n}, v_{x}\right)+\left(u^{n-1} u_{x}^{n}, v\right)=0, \quad \forall v \in V_{N}
$$

Discrete in spacial:

$$
\left(u_{N}^{n}, v_{N}\right)+v \Delta t\left(u_{N x}^{n}, v_{N x}\right)+\Delta t\left(u_{N}^{n-1} u_{N x}^{n}, v_{N}\right)=\left(u_{N}^{n-1}, v_{N}\right), \quad \forall v \in V_{N}
$$

Setting 
$u_{N}^{n}=\sum_{k=0}^{N-2}:_{k}^{n} \phi_{k}(x), \quad \forall v_{N}=\phi_{l}(x), l=1, \mathrm{~L}, N$

Then, we have

$\sum_{k=0}^{N-2}\left(\phi_{k}(x), \phi_{l}(x)\right) \stackrel{:^{n}}{u_{k}}+\Delta t \sum_{k=0}^{N-2}\left(\phi^{\prime}{ }_{k}(x), \phi^{\prime}{ }_{l}(x)\right) \stackrel{:^{n}}{u_{k}}+\Delta t I=\sum_{k=0}^{N-2}\left(\phi_{k}(x), \phi_{l}(x)\right) \dot{u}_{k}{ }^{n-1}$

Where

$$
\begin{aligned}
I & =\Delta t \sum_{k=0}^{N-2}\left[\sum_{m=0}^{N-2}\left(\phi_{m}(x) \phi^{\prime}{ }_{k}(x), \phi_{l}(x)\right):_{u_{m}}^{:-1}:{ }^{n}\right. \\
& =\Delta t D \tilde{u}_{k}^{n}
\end{aligned}
$$

Where

$$
\left.D=\left(d_{i j}\right), d_{i j}=\sum_{m=0}^{N-2}\left(\phi_{m}(x) \phi^{\prime}{ }_{k}(x), \phi_{l}(x)\right)\right)_{m}^{: n-1}
$$

Then, we have the following matrix form

$$
(M+\Delta t S+\Delta t D) \tilde{\sim}^{n}=M \tilde{\sim}^{n-1}
$$

Let the basis function $\phi_{k}(x)=L_{k}(x)-L_{k+2}(x)$, thanks to (2.11) and (1.12), we have

Lemma 2. The stiffness matrix $\mathrm{S}$ is a diagonal matrix with:

$$
s_{k k}=4 k+6, \quad k=1,2, \mathrm{~L}
$$

The mass matrix $\mathrm{M}$ is a symmetric penta-diagonal matix whose nonzero elements are

$$
m_{i j}(x)=m_{j i}(x)=\left\{\begin{array}{l}
\frac{2}{2 k+1}+\frac{2}{2 k+5}, \quad j=k ; \\
-\frac{2}{2 k+5}, \quad j=k+2 .
\end{array}\right.
$$

The proof of the above lemma can be available from[1].

Remark 5.1. Due to the basis function property, the D matrix is not easy to obtain, in this case, we have to use a galerkin numerical integral for it. We present some numerical examples using the proposed method for Burgers equation. In the following computations, the integrals involved are computed by the Legendre-Gauss-Lobatto quadrature rules. Let

$$
E_{N}(t)=\left\|u_{N}(t)-U^{N}(t)\right\|_{\omega}
$$

where $U_{N} \in V_{N}$ is the interpolation of $U$ at the Legendre-Gauss-Lobatto points $\left\{x_{j}\right\}_{j=0}^{N}$.

Example 1. Consider 1-D Burgers equation (1.1) with $v=0.01$ and its exact solution is of the form

$$
U(x, t)=\frac{1+\frac{x-t}{t+1}\left(\frac{4}{\sqrt{t+1}} e^{-\frac{(x-t)^{2}}{0.04(t+1)}}\right)}{1+\frac{4}{\sqrt{t+1}} e^{-\frac{(x-t)^{2}}{0.04(t+1)}}}
$$


This example was used by Paola Gervasio[3].

For easy to comparison, we use the same mesh size. Tablet (I) shows the error $E_{16}(t)$ at $\mathrm{t}=1$ with different time steps. Tablet (II) shows the order of accuracy for the scheme (5.5). These numerical results are listed in tablet (I) and (II).

Tablet (I): Exaple 1:Errors at $t=1$ with $\mathrm{N}=16$ using different methods.

\begin{tabular}{c|cc}
\hline Time step $\tau$ & Classical Scheme(5.1) & Proposed Scheme(5.5) \\
\hline $250^{-1}$ & $1.958 \mathrm{E}-04$ & $2.858 \mathrm{E}-06$ \\
$1000^{-1}$ & $1.957 \mathrm{E}-04$ & $1.199 \mathrm{E}-06$ \\
$4000^{-1}$ & $1.957 \mathrm{E}-04$ & $1.188 \mathrm{E}-06$ \\
\hline
\end{tabular}

Tablet (II):Example 1:Errors of the proposed scheme (5.5) with different $\tau$ and $\mathrm{N}$

\begin{tabular}{c|ccc}
\hline$\tau$ & $\mathrm{N}$ & $E_{N}(1)$ & Order \\
\hline $1 \mathrm{E}-1$ & & $1.699 \mathrm{E}-03$ & $\tau^{2.00}$ \\
$1 \mathrm{E}-2$ & 32 & $1.699 \mathrm{E}-05$ & $\tau^{2.00}$ \\
$1 \mathrm{E}-3$ & & $1.698 \mathrm{E}-07$ & $\tau^{2.00}$ \\
\hline $1 \mathrm{E}-4$ & 16 & $1.698 \mathrm{E}-09$ & $N^{-12.10}$ \\
\hline $1 \mathrm{E}-4$ & 32 & $5.181 \mathrm{E}-03$ & $N^{-16.15}$ \\
\hline
\end{tabular}

\section{Conclusions}

We have presented an efficient and accurete Legendre-Galerkin method for the Burgers equation on a bounded interval. We used a suitable scheme to instead of classical variational formulation. Our theoretical and numerical results shown that the Legendre-Galerkin approximations are stable and convergent on a bound interval with spectral accuracy.

\section{References}

[1] Jie Shen and Tao Tang, High Order Numerical Methods and Algorithms [M], April 24, 2006.

[2] Ben-Yu Guo, Spectral Methods and Their Applications, World Scientific Press [M]. Hong Kong, 1996.

[3] C.Canuto, A.Quarteroni,Approximation results for orthogonal polynomials in Sobolev spaces [M],Math. of Comp. 38, 1982.

[4] C.Canuto, M.Y.Hussaini,A.Quarteroni, T.A.Zang, Spectral Methods Fundamentals in single Domains, Springer-Verlag [M], Berlin Heidelberg 2006. 
[5] Y.Maday, S.M.Ould Kaber, E.Tadmor, Analysis of the Legendre pseudospectral approximation of a nonlinear conservation law, in[O], 1998.

[6] Jie Shen and R.Teman, Nonlinear Galerkin method using Chebyshev and Legendre polynomials I.The one-dimensional case [J], SIAM J.Numer.Anal, Vol.32,No.1,215-234, 1993.

[7] C.Mavriplis. Laguerre polynomails for infinite-domain spectral elements [J], J.Comp.Phys. 80:480-488, 1989. 